\title{
Postoperative nausea and vomiting in patients undergoing colorectal surgery within an institutional enhanced recovery after surgery protocol: comparison of two prophylactic antiemetic regimens
}

\author{
Jennifer Holder-Murray ${ }^{1}$, Stephen A Esper ${ }^{2}$, Michael L Boisen², \\ Julie Gealey ${ }^{2}$, Katie Meister ${ }^{2}$, David S Medich ${ }^{1}$, and Kathirvel Subramaniam ${ }^{2}$ \\ Departments of ${ }^{1}$ Surgery and ${ }^{2}$ Anesthesiology, University of Pittsburgh School of Medicine, Pittsburgh, PA, USA
}

Background: Enhanced recovery protocols (ERP) provide optimal perioperative care for surgical patients. Postoperative nausea and vomiting (PONV) is common after colorectal surgery (CRS). We aim to compare the efficacy of aprepitant to a cost-effective alternative, perphenazine, as components of triple antiemetic prophylaxis in ERP patients.

Methods: Patients who underwent ERP CRS at a single institution from July 2015 to July 2017 were evaluated retrospectively. Only subjects who received aprepitant (Group 1) or perphenazine (Group 2) preoperatively for PONV prophylaxis were included. Patient characteristics, simplified Apfel PONV scores, perioperative medications, and PONV incidence were compared between the groups. PONV was defined as the need for rescue antiemetics on postoperative days (POD) 0-5. Results: Five hundred ninety-seven patients underwent CRS of which 498 met the inclusion criteria. Two hundred thirty-one (46.4\%) received aprepitant and $267(53.6 \%)$ received perphenazine. The incidence of early PONV (POD 0-1) was comparable between the two groups: $44.2 \%$ in Group 1 and $44.6 \%$ in Group $2(\mathrm{P}=0.926)$. Late PONV (POD 2-5) occurred less often in Group 1 than Group 2, respectively ( $35.9 \%$ vs. $45.7 \%, \mathrm{P}=0.027$ ). After matching the groups for preoperative, procedural, and anesthesia characteristics (164 pairs), no difference in early or late PONV could be demonstrated between the groups.

Conclusions: The incidence of PONV remains high despite most patients receiving three prophylactic antiemetic medications. Perphenazine can be considered a cost-effective alternative to oral aprepitant for prophylaxis of PONV in patients undergoing CRS within an ERP.

Keywords: Aprepitant; Colectomy; Colorectal surgery; Enhanced recovery; Perphenazine; Postoperative nausea and vomiting.

Corresponding author: Kathirvel Subramaniam, M.D., MPH, FASE

Department of Anesthesiology, University of Pittsburgh Medical Center Presbyterian Hospital, University of Pittsburgh School of Medicine, 200 Lothrop Street, C Wing, Pittsburgh, PA 15213, USA

Tel: +1-4126475635, Fax: +1-4126476290,Email: subramaniamk@upmc.edu

ORCID: https://orcid.org/0000-0002-4647-1372

This work was presented at 2018 Annual Scientific Meeting of the American Society of Colon and Rectal Surgeons, May 2018, Music City Center, Nashville, TN, USA.

Received: December 16, 2018. Revised: April 3, 2019 (1st); April 25, 2019 (2nd). Accepted: May 7, 2019.

Korean J Anesthesiol 2019 August 72(4): 344-350

https://doi.org/10.4097/kja.d.18.00355

(c) This is an open-access article distributed under the terms of the Creative Commons Attribution Non-Commercial License (http://creativecommons.org/ licenses/by-nc/4.0/), which permits unrestricted non-commercial use, distribution, and reproduction in any medium, provided the original work is properly cited. 


\section{Introduction}

Enhanced recovery protocols (ERP) are designed to provide optimal perioperative care for patients undergoing surgery. Postoperative nausea and vomiting (PONV) is a common problem among patients undergoing surgery as the incidence varies from $30 \%$ to as high as $80 \%$ in various surgical procedures [1-3]. Furthermore, PONV is a contributor to increased post-anesthesia care unit (PACU) length of stay, increased hospital costs, and decreased patient satisfaction $[4,5]$.

Risk factors for PONV include sex, history of PONV or motion sickness, smoking status, age, anesthesia type, duration of anesthesia, use of volatile anesthetics including nitrous oxide, type of surgery, and opioid use. Because patient physiology and surgical type cannot be changed, current guidelines and strategies focus on the alteration of the anesthetic plan to help decrease the incidence of PONV, including the reduction or exclusion of opioids and volatile anesthetic agents $[1,3]$. Additionally, the use of prophylactic antiemetics administered preoperatively or intraoperatively is a common strategy to reduce PONV. Among the most commonly used antiemetics are $5-\mathrm{HT}_{3}$ antagonists such as ondansetron, steroids such as dexamethasone, neurokinin antagonists such as aprepitant, phenothiazine antipsychotics such as perphenazine, and anticholinergic pharmacotherapy such as scopolamine. Although it is known that the use of antiemetics is effective at preventing PONV, controversy exists regarding the best antiemetics for prevention.

At our institution, the ERP for patients undergoing colorectal surgery (CRS) included several factors including but not limited to preoperative hydration, multimodal analgesia with limitation of opioids, and the use of preoperative and intraoperative antiemetics. Aprepitant was the favored preoperative oral antiemetic utilized for patients undergoing CRS under the ERP at our center but was substituted by perphenazine because of substantially higher costs associated with aprepitant. Given the drastic difference in costs, we aimed to evaluate if there was a difference between the two medications in their antiemetic efficacy. We hypothesized that perphenazine is non-inferior to aprepitant as a preoperative prophylactic antiemetic for patients undergoing CRS within an ERP.

\section{Materials and Methods}

All patients who underwent CRS via an ERP from July 2015 to July 2017 were included. The institutional ERP is described in Table 1. Patient demographics, medication use, and other clinical variables were abstracted from the electronic medical record. Simplified Apfel scores, a measure of PONV risk, were calculated for each patient. Patients who had CRS within an ERP between July 2015 and February 2016 received oral aprepitant
$40 \mathrm{mg}$ as the standard preoperative antiemetic medication 1-2 h before surgery; the ERP was altered in February 2016 to use oral perphenazine $8 \mathrm{mg}$ as the standard preoperative antiemetic medication before surgery. Group 1 was defined as patients who received preoperative aprepitant only, and Group 2 was defined as patients who received preoperative perphenazine only. $\mathrm{Pa}$ tients who received more than one oral preoperative antiemetic were excluded from the analysis to reduce potential confounding, therefore, excluding any combination of the following medications: aprepitant, perphenazine, and scopolamine. All patients also received dexamethasone $4 \mathrm{mg}$ after induction of anesthesia and ondansetron $4 \mathrm{mg}$ before emergence for PONV prophylaxis. This study was approved by the University of Pittsburgh Institutional Review Board.

The primary outcome measure was the incidence of PONV. PONV was evaluated by the need for at least one rescue antiemetic on postoperative days (POD) 0 through 5. Early PONV was defined as the need for at least one rescue antiemetic anytime between POD 0 and 1 and late PONV was defined as the need for at least one rescue antiemetic anytime between POD 2 and 5. Ondansetron $4 \mathrm{mg}$ was used as the primary rescue antiemetic and prochlorperazine 5-10 $\mathrm{mg}$ and/or promethazine

Table 1. Colorectal ERP

\begin{tabular}{|c|c|}
\hline \multicolumn{2}{|l|}{ Preoperative protocols } \\
\hline \multirow[t]{3}{*}{ Pain management } & Acetaminophen PO \\
\hline & Gabapentin PO \\
\hline & $\begin{array}{l}\text { MS Contin PO (if intrathecal } \\
\text { contraindicated or unable to obtain) }\end{array}$ \\
\hline PONV prevention & Aprepitant or perphenazine \\
\hline Diet & Clear liquids until $3 \mathrm{~h}$ prior to surgery \\
\hline \multicolumn{2}{|c|}{ Intraoperative protocols } \\
\hline \multirow[t]{2}{*}{ Pain management } & Intrathecal morphine injection \\
\hline & Ketoralac IV, at surgeon discretion \\
\hline \multirow[t]{2}{*}{ PONV prevention } & Dexamethasone IV \\
\hline & Ondansetron IV \\
\hline \multirow[t]{3}{*}{ Anesthesia } & General \\
\hline & Ketamine IV drip \\
\hline & Lidocaine IV drip \\
\hline Fluid management & Goal-directed IV fluid therapy \\
\hline \multicolumn{2}{|l|}{ Postoperative protocols } \\
\hline \multirow[t]{5}{*}{ Pain management } & Lidocaine IV drip for $24 \mathrm{~h}$ \\
\hline & Acetaminophen IV transitioned to PO \\
\hline & Ketoralac IV transitioned to ibuprofen PO \\
\hline & Oxycodone, prn \\
\hline & Hydromorphone IV, prn breakthrough pain \\
\hline \multirow[t]{2}{*}{ PONV treatment } & Ondansetron IV, prn \\
\hline & Additional antiemetic, prn \\
\hline Fluid management & Stop IV fluids on POD 1 unless ileostomy \\
\hline Diet & $\begin{array}{l}\text { Clear liquids on POD } 0 \text {, advanced diet on } \\
\text { POD } 1\end{array}$ \\
\hline
\end{tabular}

ERP: enhanced recovery protocols, PO: per os, MS Contin: morphine sulfate extended release, PONV: postoperative nausea and vomiting, IV: intravenous, POD: postoperative day. 
12.5-25 mg were utilized in PONV refractory to ondansetron. Each antiemetic was evaluated for their incidence and frequency of use. Postoperative use of opioid medications (fentanyl, oxycodone, hydromorphone, and morphine) was noted for both groups. Each opioid pain medication dose was converted into intravenous morphine equivalents (IV ME) to evaluate total opioid consumption. As prolonged postoperative ileus may alter the incidence of PONV, we noted and compared the incidence between the two groups. Continuous parameters were analyzed using the Mann-Whitney $U$ test, and all dichotomous variables were analyzed by $\mathrm{Chi}^{2}$ test. In order to compare patients with similar patient characteristics, anesthesia technique, and other operative parameters, Group 1 patients were propensity matched to Group 2 patients for an additional analysis. Matching was done using a predictive score derived from logistic regression with a caliper distance of less than 0.05 , yielding 164 matched pairs (1: 1 matching). Baseline characteristics and PONV outcomes were then compared between the matched groups. An alpha level of 0.05 was used to determine statistical significance. SPSS (IBM Corp., USA) version 25.0 was used for statistical analysis.

\section{Results}

A total of 597 patients underwent CRS during the study period. Sixty-nine patients received neither aprepitant nor perphenazine, 22 patients received transdermal scopolamine patch, eight patients received both perphenazine and aprepitant; all of these patients were excluded from the study. Therefore, 498 met the inclusion criteria for our study. Group 1 consisted of 231 (46.4\%) patients who received aprepitant only while Group 2 consisted of 267 (53.6\%) who received perphenazine only as oral preoperative antiemetic prophylaxis before surgery.
Patients in Group 1 received a significantly higher dose of intraoperative IV ketamine $(\mathrm{P}<0.001)$ and a trend toward increased use of total intravenous anesthesia (TIVA) $(\mathrm{P}=0.06)$ than patients in Group 2. However, TIVA was used in very few patients in both groups: 13 patients in Group 1 (5.6\%) and 6 patients (2.2\%) in Group 2. All others received inhalational anesthesia for maintenance anesthesia during the surgery. Group 2 patients had a trend toward a higher frequency of smoking history $(\mathrm{P}=0.071)$ and received spinal opioids more often $(\mathrm{P}=$ 0.001) than Group 1 patients (Table 2). The distribution of simplified Apfel scores within the two groups was comparable (Table 3).

As baseline patient characteristics and anesthesia techniques (spinal opioids, smoking history, TIVA, and midazolam) can influence the incidence of PONV, propensity matching was performed to obtain patients matched for those variables. We did not attempt to match patients for IV ketamine dose, as a protocol change in ketamine dosage $(0.6 \mathrm{mg} / \mathrm{kg} / \mathrm{h}$ to $0.4 \mathrm{mg} / \mathrm{kg} / \mathrm{h})$ occurred at the time of the antiemetic protocol change (aprep-

Table 3. Simplified Apfel Scores

\begin{tabular}{cccccc}
\hline \multirow{2}{*}{$\begin{array}{c}\text { Apfel } \\
\text { score }\end{array}$} & \multicolumn{2}{c}{ Unmatched cohort } & & \multicolumn{2}{c}{ Matched cohort } \\
\cline { 2 - 3 } \cline { 5 - 6 } & $\begin{array}{c}\text { Group 1 } \\
(\mathrm{n}=231)\end{array}$ & $\begin{array}{c}\text { Group 2 } \\
(\mathrm{n}=267)\end{array}$ & & $\begin{array}{c}\text { Group 1 } \\
(\mathrm{n}=164)\end{array}$ & $\begin{array}{c}\text { Group 2 } \\
(\mathrm{n}=164)\end{array}$ \\
\hline 0 & $7(3.0)$ & $13(4.9)$ & & $7(3.2)$ & $5(3.0)$ \\
1 & $104(45.0)$ & $104(40.0)$ & & $72(47.9)$ & $62(43.6)$ \\
2 & $100(43.3)$ & $113(42.3)$ & & $72(42.0)$ & $78(44.7)$ \\
3 & $20(8.7)$ & $36(13.5)$ & & $13(6.9)$ & $19(7.4)$ \\
4 & 0 & $1(0.4)$ & & 0 & 0 \\
\hline
\end{tabular}

Values expressed as $\mathrm{n}(\%)$; $\mathrm{P}$ value $=0.232$ for unmatched cohort, and $\mathrm{P}=0.485$ for matched cohort by Chi-square test for comparing multiple proportions.

Table 2. Baseline Characteristics

\begin{tabular}{|c|c|c|c|c|c|c|}
\hline \multirow{2}{*}{ Variable } & \multicolumn{3}{|c|}{ Unmatched cohort } & \multicolumn{3}{|c|}{ Matched cohort } \\
\hline & Group $1(\mathrm{n}=231)$ & Group $2(n=267)$ & $P$ value & Group $1(\mathrm{n}=164)$ & Group $2(\mathrm{n}=164)$ & $P$ value \\
\hline Age (yr) & $54.0(37.0-66.0)$ & $54.0(40.0-67.0)$ & 0.292 & $57.0(42.5-68.8)$ & $51.0(30.0-65.0)$ & 0.739 \\
\hline Sex, M & $107(46.3 \%)$ & $125(46.8 \%)$ & 0.912 & $77(46.9 \%)$ & $72(43.9 \%)$ & 0.579 \\
\hline Current smoking & $31(13.4 \%)$ & $52(19.4 \%)$ & 0.071 & $23(14 \%)$ & $26(15.8 \%)$ & 0.642 \\
\hline Admit weigh $(\mathrm{kg})$ & $77.0(61.0-88.0)$ & $75.0(64.0-88.0)$ & 0.429 & $73.0(61.0-88.0)$ & $75.0(61.1-85.8)$ & 0.144 \\
\hline Open procedure & $90(39.0 \%)$ & $103(38.6 \%)$ & 0.930 & $67(40.8 \%)$ & $67(40.8 \%)$ & 1.000 \\
\hline Length of surgery (min) & $180(93-243)$ & $153(95-245)$ & 0.807 & $185.7(94.5-259.6)$ & $152.7(90.6-213.9)$ & 0.668 \\
\hline Spinal opioids & $162(70.1 \%)$ & $220(82.4 \%)$ & 0.001 & $129(78.7 \%)$ & $125(76.2 \%)$ & 0.597 \\
\hline Intraoperative ketamine (mg) & $155.4(106.8-219.1)$ & $122.7(88.2-165.1)$ & $<0.001$ & $139.6(95.0-199.7)$ & $131.8(92.4-184.4)$ & $<0.001$ \\
\hline Intraoperative midazolam (mg) & $1.0(0.0-2.0)$ & $1.0(0.0-2.0)$ & 0.051 & $1.0(0.0-2.0)$ & $1.0(0.0-2.0)$ & 0.544 \\
\hline $\begin{array}{l}\text { Parenteral and oral opioids, } \\
\text { (preoperative + intraoperative) }\end{array}$ & $25(10.8 \%)$ & $31(11.6 \%)$ & 0.781 & $12(7.3 \%)$ & $19(11.6 \%)$ & 0.186 \\
\hline Total intravenous anesthesia & $13(5.6 \%)$ & $6(2.2 \%)$ & 0.050 & $1(0.6 \%)$ & $3(1.8 \%)$ & 0.615 \\
\hline
\end{tabular}

Values are presented as number (\%) for categorical and as median (IQR 25\%-75\%) for continuous variables. 
Table 4. PONV Incidence and Severity

\begin{tabular}{|c|c|c|c|c|c|c|}
\hline \multirow[b]{2}{*}{ Variable } & \multicolumn{3}{|c|}{ Unmatched cohort } & \multicolumn{3}{|c|}{ Matched cohort } \\
\hline & $\begin{array}{c}\text { Group 1 } \\
(\mathrm{n}=231)\end{array}$ & $\begin{array}{c}\text { Group } 2 \\
(\mathrm{n}=267)\end{array}$ & $P$ value & $\begin{array}{c}\text { Group 1 } \\
(\mathrm{n}=164)\end{array}$ & $\begin{array}{c}\text { Group 2 } \\
(\mathrm{n}=164)\end{array}$ & P value \\
\hline Early PONV (POD 0-1) & $102(44.2 \%)$ & $119(44.6 \%)$ & 0.926 & $73(44.5 \%)$ & $72(43.9 \%)$ & 0.911 \\
\hline Late PONV (POD 2-5) & $83(35.9 \%)$ & $122(45.7 \%)$ & 0.027 & $60(36.5 \%)$ & $74(45.1 \%)$ & 0.116 \\
\hline Ondansetron (POD 0-5) & $127(54.9 \%)$ & $159(59.5 \%)$ & 0.303 & $91(55.5 \%)$ & $95(57.9 \%)$ & 0.656 \\
\hline Prochlorperazine (POD 0-5) & $26(11.2 \%)$ & $37(13.9 \%)$ & 0.384 & $18(10.9 \%)$ & $22(13.4 \%)$ & 0.500 \\
\hline Promethazine (POD 0-5) & $9(3.9 \%)$ & $9(3.4 \%)$ & 0.754 & $6(3.6 \%)$ & $6(3.6 \%)$ & 1.000 \\
\hline Frequency of ondansetron doses (Early PONV) & $0(0-1)$ & $0(0-1)$ & 0.944 & $0(0-1)$ & $0(0-1)$ & 0.886 \\
\hline Frequency of ondansetron doses (Late PONV) & $0(0-1)$ & $0(0-2)$ & 0.031 & $0(0-1.75)$ & $0(0-2)$ & 0.071 \\
\hline
\end{tabular}

Values are presented as number (\%) or median (IQR 25\%-75\%). PONV: postoperative nausea and vomiting, POD: postoperative day.

Table 5. Postoperative Opioid Requirements

\begin{tabular}{|c|c|c|c|c|c|c|}
\hline \multirow[b]{2}{*}{ Variable } & \multicolumn{3}{|c|}{ Unmatched cohort } & \multicolumn{3}{|c|}{ Matched cohort } \\
\hline & $\begin{array}{l}\text { Group 1 } \\
(\mathrm{n}=231)\end{array}$ & $\begin{array}{c}\text { Group 2 } \\
(\mathrm{n}=267)\end{array}$ & $P$ value & $\begin{array}{c}\text { Group 1 } \\
(\mathrm{n}=164)\end{array}$ & $\begin{array}{c}\text { Group 2 } \\
(\mathrm{n}=164)\end{array}$ & $P$ value \\
\hline IV ME (POD 0-1) & $15.5(2.9-39.0)$ & $21.9(6.3-43.0)$ & 0.047 & $19.3(5.1-38.3)$ & $17.9(5.8-42.0)$ & 0.110 \\
\hline IV ME (POD 2-5) & $15(0-45)$ & $20(0-52.7)$ & 0.334 & $15(2.5-39.7)$ & $15.8(0.0-43.5)$ & 0.535 \\
\hline IV ME (POD 0-5) & $33.2(8.0-85.2)$ & $46.9(14.0-94.0)$ & 0.087 & $37.6(13.1-80.1)$ & $35.5(9.45-86.8)$ & 0.174 \\
\hline Oxycodone PO, mg (POD 0-5) & $55(5-132.5)$ & $70(15-140)$ & 0.258 & $57.5(10-119.4)$ & $55(10-130)$ & 0.747 \\
\hline Hydromorphone IV, mg (POD 0-5) & $0.8(0-2.4)$ & $1.2(0-2.8)$ & 0.110 & $1(0-2.5)$ & $0.95(0-2.6)$ & 0.031 \\
\hline Number of patients required any opioids (POD $0-5$ ) & $222(96.1 \%)$ & $265(99.3 \%)$ & 0.017 & $139(84.8 \%)$ & $147(89.6 \%)$ & 0.186 \\
\hline Number of patients required IV fentanyl (POD 0-5) & $15(6.4 \%)$ & $10(3.7 \%)$ & 0.161 & $8(4.9 \%)$ & $4(2.4 \%)$ & 0.378 \\
\hline
\end{tabular}

Values are presented as number (\%) or median (IQR 25\%-75\%). ME: intravenous morphine equivalents, POD: postoperative day, PO: per oral, IV: intravenous.

itant to perphenazine). After matching, only the intraoperative ketamine dose remained significantly different between the two groups; all other factors were comparable and statistically similar.

The incidence of early PONV (POD 0-1) was comparable between unmatched and matched patients with $44.2 \%$ in Group 1 and $44.6 \%$ in Group 2 ( $\mathrm{P}=0.926)$, and $44.5 \%$ and $43.9 \%$ ( $\mathrm{P}=$ 0.911 , respectively. Prophylaxis with aprepitant significantly reduced the incidence of late PONV (POD 2-5) when compared to perphenazine prophylaxis $35.9 \%$ in Group 1 vs. $45.7 \%$ in Group 2, P = 0.027) in the unmatched cohort. The frequency of ondansetron administration during POD 2-5 was also significantly less with aprepitant prophylaxis in the unmatched cohort (Table 4). For matched pairs, the incidence of both early and late PONV was not significantly different between the two groups. The frequency of each postoperative antiemetic (ondansetron, prochlorperazine, and promethazine) administration for POD 0-5 was comparable between the matched patients (Table 4). Of note, postoperative ileus rates, defined as nasogastric tube insertion or NPO at POD 4, were similar between both unmatched (39.8\% vs. $37.8 \%, \mathrm{P}=0.692)$ and matched patients $(42.7 \%$ and $37.8 \%, \mathrm{P}=0.368)$.
Although there was a significant difference in the utilization of IV hydromorphone between matched patients, overall opioid consumption measured by IV ME and the number of patients requiring opioid analgesics were found to be similar (Table 5).

The cost of aprepitant at our institution is $\$ 130$ vs. $\$ 2$ for perphenazine. Therefore, assuming approximately 250 major abdominal CRS ERP surgeries at our single institution in one year, substitution of perphenazine for aprepitant results in a cost savings of $\$ 32,000$ per year.

\section{Discussion}

The incidence of PONV after CRS varies between 12 and $47 \%$ in clinical trials [6-8]. These variations could be explained by the type of surgical procedure, anesthesia medications utilized, the methods used to evaluate PONV, the antiemetic prophylaxis protocol, type of postoperative analgesia, and the perioperative feeding methods utilized. Perioperative use of opioids is often considered as a risk factor for PONV and ERP in CRS often utilize multimodal analgesic techniques to minimize opioid analgesics. A meta-analysis of randomized clinical trials utilizing ERP pain management pathways has reported a 
decrease in the incidence of PONV by $18 \%$ [9]. In a multicenter clinical trial of 300 patients undergoing various colorectal procedures, non-opioid analgesia was utilized in $78 \%$ of patients and epidural analgesia in $38 \%$ of patients [10]. The incidence of PONV was very low (12\%) considering the antiemetic prophylaxis was administered only to $27 \%$ of patients in their study [10].

We report a higher incidence of PONV $(55 \%-60 \%)$ in this study utilizing an ERP for CRS even with triple antiemetic prophylaxis (dexamethasone and ondansetron with either perphenazine or aprepitant). The ERP in this study utilized liberation of fasting, early feeding, and early mobilization, all of which have been associated with decreasing PONV. Minimally invasive surgical techniques whenever feasible were utilized with approximately $60 \%$ of patients receiving laparoscopic surgery, which may increase PONV rates as laparoscopy and the creation of pneumoperitoneum is a known risk factor for PONV Intrathecal morphine, intravenous lidocaine and ketamine, acetaminophen, and non-steroidal anti-inflammatory drugs were utilized for analgesia. Postoperative rescue opioid analgesia consisted of oral oxycodone and intravenous hydromorphone in titrated doses with patient-controlled analgesia only reserved for refractory pain. Intraoperative opioids are avoided except in patients with contraindications for intrathecal morphine. The high incidence of PONV could be explained by several factors. First, neuraxial opioids are well known to cause significant PONV [11]. In a meta-analysis of twenty-eight placebo-controlled studies $(\mathrm{n}=1,314$ patients) utilizing intrathecal morphine, a total of 790 patients received intrathecal morphine and 524 patients received a placebo. There were statistically significant increases in nausea (RR $1.3,95 \%$ CI 1.1 to $1.5 ; 24$ RCTs), vomiting (RR $1.6,95 \%$ CI 1.1 to 2.2; 19 RCTs), and pruritus (RR 2.0, 95\% CI 1.6 to $2.4 ; 25$ RCTs) with intrathecal morphine compared with placebo [11]. Second, ketamine's opioid-sparing analgesic benefits are described in the literature, though the effect of opioid sparing on opioid-related side effects such as PONV is still controversial and a known side effect of ketamine is increased PONV [12-14]. As ketamine is the mainstay of our ERP, this may have contributed to the high incidence of PONV. Third, we utilized mainly inhalational anesthesia except in very high-risk patients for PONV. Total intravenous anesthesia (TIVA) was used only in 19 (3.8\%) patients in this study and the use of TIVA with propofol could have reduced the incidence of PONV [15]. Finally, although intraoperative opioid use could be avoided in almost $90 \%$ of patients, the use of postoperative opioids was still frequent with more than $95 \%$ of patients receiving some rescue opioids during the study period. The use of continuous catheter analgesia with local anesthetics (epidural or peripheral nerve blocks) could have further reduced the need for opioids and thus potentially the PONV rate.

The incidence of late PONV (POD 2-5 days) was also higher $(35 \%-45 \%)$ and the concern of delayed PONV was addressed recently $[16,17]$. Although there are abundant data on PONV occurrence until $24 \mathrm{~h}$ postoperatively, delayed PONV and post-discharge nausea and vomiting after ambulatory surgery deserve further investigation [18-20]. One recent large multicenter pragmatic study (DREAMS trial) involved 1350 participants and evaluated PONV and rescue medication use for $120 \mathrm{~h}$ postoperatively in major bowel surgery [21]. The incidences of antiemetic use at 24,72 , and $120 \mathrm{~h}$ were $39 \%, 52 \%$, and $41 \%$ in the dexamethasone group and $52 \%, 63 \%$, and $42 \%$ in the standard of care group without dexamethasone. The incidence was quite similar to what we found in our study. Late PONV can be more relevant in colorectal procedures where there is persistence of gastrointestinal irritation and insults even after the anesthetic effects wear off. For example, the persistence of paralytic ileus (defined as NPO on POD 4 or new postoperative insertion of a nasogastric tube) was present in at least one-third of the patients in both of our groups, and likely contributed to the higher rates of late PONV seen throughout our study. Additional measures to decrease postoperative ileus like utilization of continuous epidural analgesia, peripheral nerve blocks, alvimopan, and optimization of goal-directed fluid therapy regimens might improve the late PONV in this patient population.

Ondansetron-a 5- $\mathrm{HT}_{3}$ antagonist-and dexamethasone are recommended as combination antiemetic prophylaxis in patients at high risk for PONV such as laparoscopic CRS [22]. Since patients undergoing CRS are at higher risk for PONV, we decided to add a third drug for antiemetic prophylaxis. Aprepitant, a neurokinin (NK-3) antagonist, has shown better efficacy in producing complete response against PONV when compared with ondansetron in patients at high risk for PONV and is considered the drug of choice for PONV refractory to other drugs [23-26]. Aprepitant also has the advantage of longer half-life compared to $5-\mathrm{HT}_{3}$ antagonists [27]. However, as aprepitant is more expensive compared to other antiemetics, we replaced aprepitant with perphenazine, a phenothiazine used for treating depression but also nausea and vomiting [28]. A systematic review of 11 clinical trials $(n=2,081)$ has shown that perphenazine significantly reduced the incidence of PONV (RR 0.50; 95\% CI: 0.37-0.67) compared to placebo and was equally efficacious compared to other antiemetic drugs [29]. Adverse events such as extrapyramidal symptoms were rare with the use of perphenazine [30]. While aprepitant is a relatively new drug with its efficacy proven from well-conducted clinical trials, perphenazine is an older, underappreciated drug with its studies predominantly conducted before 2000 and no studies have compared the two.

To date, there is no study that compares the antiemetic efficacy of perphenazine and aprepitant in an ERP patient population. There was no difference in the need for rescue antiemetics in the early postoperative period (POD 0-1) but there was a trend 
towards less PONV in the late postoperative period with aprepitant although the difference did not reach statistical significance in the matched cohort. This marginal and questionable late benefit could be related to aprepitant's longer half-life, which needs to be studied in future prospective studies. This could also be accounted for in patient factors such as infection, which was not measured in our study. Nevertheless, the incidence of PONV is very high necessitating additional strategies for prevention of PONV apart from pharmacological prophylaxis in this population as described above.

Several limitations should be considered while interpreting the results of the study. First, this is a single center retrospective study and the antiemetic efficacy was mainly evaluated by the requirement of rescue antiemetics. The incidence of nausea could not be separated from the incidence of vomiting from the documentation. Second, the predicted risk of PONV could not be reliably estimated retrospectively as the calculated Apfel scores from existing records may underestimate the actual risk without additional documentation of patient reported factors. Third, compliance with ERP components was not measured in this study, which could have affected the incidence of PONV. Fourth, oral antiemetics are typically recommended to be given 1-2 $\mathrm{h}$ before surgery. Though our practice is for administration in this interval of time, the timing of administration for perphenazine and aprepitant was not monitored and could have affected antiemetic efficacy and results. Finally, it is not possible to conclude on the antiemetic efficacy of orally administered drugs before surgery unless we include a group without both of these drugs (a placebo group). Nonetheless, we can address the inferiority of two medications.

Despite the above limitations of this retrospective study, we present a comparative analysis of two prophylactic antiemetic medications as components of a triple antiemetic prophylaxis regimen in a large ERP population of CRS patients. The incidence of PONV remained high despite triple prophylaxis and ERP measures (opioid restricted perioperative pain management), possibly related to surgical factors like postoperative ileus. We demonstrate that perphenazine has a similar efficacy as aprepitant for prophylaxis of PONV in this population and can be considered as a cost-effective alternative. Other strategies of PONV prevention such as total intravenous anesthesia and local anesthesia based regional analgesic infusions should be considered for these patients in addition to pharmacological prophylaxis given the overall high rate of PONV.

\section{Conflicts of Interest}

No potential conflict of interest relevant to this article was reported.

\section{Author Contributions}

Jennifer Holder-Murray (Conceptualization; Investigation; Methodology; Supervision; Validation; Writing - original draft; Writing - review \& editing)

Stephen A Esper (Conceptualization; Investigation; Supervision; Validation; Writing - original draft; Writing - review \& editing) Michael L Boisen (Investigation; Validation; Writing - original draft; Writing - review \& editing)

Julie Gealey (Data curation; Formal analysis; Project administration; Validation; Writing - original draft; Writing - review \& editing)

Katie Meister (Conceptualization; Data curation; Investigation; Project administration; Writing - review \& editing)

David S Medich (Investigation; Project administration; Supervision; Validation; Writing - original draft; Writing - review \& editing)

Kathirvel Subramaniam (Data curation; Formal analysis; Investigation; Methodology; Project administration; Resources; Software; Supervision; Validation; Visualization; Writing - original draft; Writing - review \& editing)

\section{ORCID}

Jennifer Holder-Murray, https://orcid.org/0000-0003-0030-9297 Stephen A Esper, https://orcid.org/0000-0003-4839-8030

Michael L Boisen, https://orcid.org/0000-0002-9320-4795

Julie Gealey, https://orcid.org/0000-0002-0354-2775

Katie Meister, https://orcid.org/0000-0002-4027-7405

David S Medich, https://orcid.org/0000-0001-5008-401X

Kathirvel Subramaniam, https://orcid.org/0000-0002-4647-1372

\section{References}

1. Sinclair DR, Chung F, Mezei G. Can postoperative nausea and vomiting be predicted? Anesthesiology 1999; 91: 109-18.

2. Koivuranta M, Läärä E, Snåre L, Alahuhta S. A survey of postoperative nausea and vomiting. Anaesthesia 1997; 52: 443-9.

3. Apfel CC, Läärä E, Koivuranta M, Greim CA, Roewer N. A simplified risk score for predicting postoperative nausea and vomiting: conclusions from cross-validations between two centers. Anesthesiology 1999; 91: 693-700.

4. Macario A, Weinger M, Carney S, Kim A. Which clinical anesthesia outcomes are important to avoid? The perspective of patients. Anesth 
Analg 1999; 89: 652-8.

5. Gan T, Sloan F, Dear Gde L, El-Moalem HE, Lubarsky DA. How much are patients willing to pay to avoid postoperative nausea and vomiting? Anesth Analg 2001; 92: 393-400.

6. Park HE, Kim MK, Kang WK. Efficacy and safety of ramosetron injection for nausea and vomiting in colorectal-cancer patients undergoing a laparoscopic colectomy: a randomized, double-blind, comparative study. Ann Coloproctol 2018; 34: 36-41.

7. Barclay KL, Zhu YY, Tacey MA. Nausea, vomiting and return of bowel function after colorectal surgery. ANZ J Surg 2015; 85: 823-8.

8. Kong SK, Onsiong SM, Chiu WK, Li MK. Use of intrathecal morphine for postoperative pain relief after elective laparoscopic colorectal surgery. Anaesthesia 2002; 57: 1168-73.

9. Chemali ME, Eslick GD. A meta-analysis: postoperative pain management in colorectal surgical patients and the effects on length of stay in an enhanced recovery after surgery (ERAS) setting. Clin J Pain 2017; 33: 87-92.

10. Ramírez JM, Blasco JA, Roig JV, Maeso-Martínez S, Casal JE, Esteban F, et al. Enhanced recovery in colorectal surgery: a multicentre study. BMC Surg 2011; $11: 9$.

11. Gehling M, Tryba M. Risks and side-effects of intrathecal morphine combined with spinal anaesthesia: a meta-analysis. Anaesthesia 2009; 64: 643-51.

12. Subramaniam K, Subramaniam B, Steinbrook RA. Ketamine as adjuvant analgesic to opioids: a quantitative and qualitative systematic review. Anesth Analg 2004; 99: 482-95.

13. Lonjaret L, Bataille B, Gris C, Fourcade O, Minville V. Ketamine and postoperative nausea and vomiting: role of the morphine-sparing effect. J Clin Anesth 2012; 24: 601-2.

14. Minoshima R, Kosugi S, Nishimura D, Ihara N, Seki H, Yamada T, et al. Intra- and postoperative low-dose ketamine for adolescent idiopathic scoliosis surgery: a randomized controlled trial. Acta Anaesthesiol Scand 2015; 59: 1260-8.

15. Ziemann-Gimmel P, Goldfarb AA, Koppman J, Marema RT. Opioid-free total intravenous anaesthesia reduces postoperative nausea and vomiting in bariatric surgery beyond triple prophylaxis. Br J Anaesth 2014; 112: 906-11.

16. Brookes CD, Turvey TA, Phillips C, Kopp V, Anderson JA. Postdischarge nausea and vomiting remains frequent after le fort i osteotomy despite implementation of a multimodal antiemetic protocol effective in reducing postoperative nausea and vomiting. J Oral Maxillofac Surg 2015; 73: 1259-66.

17. Apfel CC, Philip BK, Cakmakkaya OS, Shilling A, Shi YY, Leslie JB, et al. Who is at risk for postdischarge nausea and vomiting after ambulatory surgery? Anesthesiology 2012; 117: 475-86.

18. Skolnik A, Gan TJ. Update on the management of postoperative nausea and vomiting. Curr Opin Anaesthesiol 2014; $27: 605-9$.

19. Welliver M. Postdischarge nausea and vomiting should be a greater concern. Gastroenterol Nurs 2018; 41: 356-60.

20. Moon YE. Postoperative nausea and vomiting. Korean J Anesthesiol 2014; 67: 164-70.

21. DREAMS Trial Collaborators and West Midlands Research Collaborative. DREAMS Trial Collaborators and West Midlands Research Collaborative. BMJ 2017; 357: j1455.

22. Gan TJ, Diemunsch P, Habib AS, Kovac A, Kranke P, Meyer TA, et al. Consensus guidelines for the management of postoperative nausea and vomiting. Anesth Analg 2014; 118: 85-113.

23. Milnes V, Gonzalez A, Amos V. Aprepitant: a new modality for the prevention of postoperative nausea and vomiting: an evidence-based review. J Perianesth Nurs 2015; 30: 406-17.

24. Diemunsch P, Apfel C, Gan TJ, Candiotti K, Philip BK, Chelly J, et al. Preventing postoperative nausea and vomiting: post hoc analysis of pooled data from two randomized active-controlled trials of aprepitant. Curr Med Res Opin 2007; 23: 2559-65.

25. Diemunsch P, Gan TJ, Philip BK, Girao MJ, Eberhart L, Irwin MG, et al. Single-dose aprepitant vs ondansetron for the prevention of postoperative nausea and vomiting: a randomized, double-blind phase III trial in patients undergoing open abdominal surgery. $\mathrm{Br} \mathrm{J}$ Anaesth 2007; 99: 202-11.

26. Gan TJ, Apfel CC, Kovac A, Philip BK, Singla N, Minkowitz H, et al. A randomized, double-blind comparison of the NK1 antagonist, aprepitant, versus ondansetron for the prevention of postoperative nausea and vomiting. Anesth Analg 2007; 104: 1082-9.

27. Singh PM, Borle A, Rewari V, Makkar JK, Trikha A, Sinha AC, et al. Aprepitant for postoperative nausea and vomiting: a systematic review and meta-analysis. Postgrad Med J 2016; 92: 87-98.

28. Williams BA, Orebaugh SL, Kentor ML. Post-operative nausea and vomiting prevention with perphenazine: long overdue. Eur J Anaesthesiol 2011; 28: 141-2.

29. Schnabel A, Eberhart LH, Muellenbach R, Morin AM, Roewer N, Kranke P. Efficacy of perphenazine to prevent postoperative nausea and vomiting: a quantitative systematic review. Eur J Anaesthesiol 2010; 27: 1044-51.

30. Henao JP, Peperzak KA, Lichvar AB, Orebaugh SL, Skledar SJ, Pippi MA, et al. Extrapyramidal symptoms following administration of oral perphenazine 4 or 8mg: an 11-year retrospective analysis. Eur J Anaesthesiol 2014; 31: 231-5. 